\title{
Effect of Hemoglobin on the Prognosis of Patients with Advanced Cancer in Palliative Care Settings
}

xinghe liao

Fudan University Shanghai Cancer Center

Cihang Bao

Fudan University

Minghui Liu

Fudan University Shanghai Cancer Center

Menglei Chen

Fudan University Shanghai Cancer Center

Xiaoli Gu

Fudan University Shanghai Cancer Center

Zhe Zhang

Fudan University Shanghai Cancer Center

Jin Zhang

Fudan University Shanghai Cancer Center

Chenyue Zhang

Fudan University Shanghai Cancer Center

Weiwei Zhao

Fudan University Shanghai Cancer Center

Wenwu Cheng ( $\nabla$ cwwxxm@sina.com )

Fudan University Shanghai Cancer Center

\section{Research Article}

Keywords: hemoglobin, prognostic value, advanced cancer, palliative care

Posted Date: September 23rd, 2021

DOI: https://doi.org/10.21203/rs.3.rs-850589/v1

License: (1) This work is licensed under a Creative Commons Attribution 4.0 International License.

Read Full License 


\section{Abstract}

Background: Hemoglobin is a prognostic factor for many cancer patients. However, its effect on the prognosis of patients with advanced cancer receiving palliative treatment is still unclear.

Objective: The aim of this study was to assess whether hemoglobin can be used as a prognostic indicator for patients with advanced cancer receiving palliative treatment.

Methods: From July 2013 to October 2015, 368 consecutive patients were treated in the palliative treatment center of the Shanghai Cancer Center of Fudan University. The data for 105 patients were extended in the follow-up. The cut-off value selected for hemoglobin was $100 \mathrm{~g} / \mathrm{L}$.

Results: The median survival time of patients with low hemoglobin was shorter than that of patients with high hemoglobin (41 days vs. 84 days). In the follow-up readmission cohort $(n=105)$, the median survival time for patients with high hemoglobin $(\mathrm{HHb} \rightarrow \mathrm{HHb})$ was 3.44 times longer than that of patients with low hemoglobin $(\mathrm{HHb} \rightarrow \mathrm{LHb}$ ). Thus, both low hemoglobin and decreased hemoglobin were identified as independent prognostic factors for poor prognosis.

Conclusions: In palliative treatment, hemoglobin can be used as a stratification factor to determine the prognosis of advanced cancer patients.

\section{Introduction}

Cancer is a major public health problem and the number of cancer patients will increase worldwide, leading to serious health consequences ${ }^{[1]}$. Advanced cancer poses a great challenge to treatment and is a common cause of death ${ }^{[1]}$. Palliative care is an essential part of overall cancer treatment ${ }^{[2]}$. In palliative care, clinicians need to predict the life expectancy of patients with advanced cancer because determining the possibility of impending death aids doctors in clinical decision-making to help patients and their families prepare for the future ${ }^{[3,4]}$. In the past few decades, several prognostic models have been proposed. The most commonly used prognostic model in palliative treatment is the Clinical Prediction of Survival; however, its inherent non-repeatability limits its accuracy and clinical application ${ }^{[5]}$. Therefore, more accurate and easily detected indicators to predict the prognosis of patients with advanced cancer are greatly needed.

Hemoglobin $(\mathrm{HB})$ is widely used in clinical examination ${ }^{[6]}$. Low hemoglobin $(\mathrm{HB})$ levels are common in cancer patients (approximately $30-77 \%)^{[7]}$. Although the number of studies exploring the prognostic role of $\mathrm{Hb}$ levels has been increasing, the results are inconsistent and usually based on small samples. Few studies have reported the effect of low hemoglobin on the survival of patients with advanced cancer receiving palliative treatment.

\section{Materials And Methods}




\section{Data collection and study cohort}

Consecutive inpatients who were treated at the Department of palliative care, Shanghai Cancer Center (fuscc), Fudan University, Shanghai, China, were retrospectively enrolled between July 2013 and October 2015. We collected data on patients' age, sex, comorbidities, smoking status, family history, primary tumor location, tumor stage, nutritional status, and physical status (Eastern Cooperative Oncology Group, ECOG score) were obtained from the patient medical records. Hemoglobin levels were measured 1-3 days before the start of palliative care. According to the ESPEN guidelines for nutrition screening, an unintentional weight loss of $>5 \%$ in the previous three months or a food intake below $75 \%$ of the normal requirement in the preceding week were considered an abnormal nutritional status ${ }^{[8]}$. The presence of concomitant disease was defined as self-reported cardiac disease, hypertension, diabetes, or any cerebrovascular disease.

Two cohorts of patients were identified in our study ${ }^{[9]}$. Palliatively treated patients with malignancies in stages III-IV were classified as cohort 1, and the completeness of patient data was guaranteed. Patients in cohort 1 were classified after readmission to cohort 2. The effect of HB changes on OS was assessed. The last follow-up date was December 2015. The study was approved by the ethics committee of fuscc. Informed consent was waived due to the retrospective nature of the study.

\section{Statistics analysis}

Data are presented as the mean \pm standard deviation (SD) for continuous variables and as totals and frequencies for categorical variables. The distributions of the clinicopathological features were tested using the Wilcoxon sum rank test, chi-squared test, or Fisher's exact test, as appropriate. The KaplanMeier method was used to plot the survival curves and the log-rank test was conducted to test differences between groups. Cox proportional hazards models were employed to estimate the magnitude of the association between the clinicopathological features and OS. X-tile version 3.6.1 (Yale University, New Haven, CT, USA) was used to determine the optimal cut-off value. All tests were two-sided, and $P$ values less than 0.05 were considered statistically significant. Statistical analyses were performed with SPSS (version 19.0), and R software (version 3.5.3) was used to draw survival curves.

\section{Results}

\section{Patient characteristics}

A total of 368 qualified patients were identified for cohort 1 in this study. Of the 368 patients, 105 had readmission data and were selected for cohort 2. There were 201 males (54.62\%) and 167 females (45.38\%) in cohort 1. Most patients had stage IV disease (92.12\%). In the classification of tumors, gastrointestinal tumors $(189,51.36 \%)$ accounted for the largest proportion of patients, while head and neck cancer $(17,4.62 \%)$ accounted for a relatively small proportion. Most patients received the best supportive care $(275,74.73 \%)$. There was no significant difference in the number of patients with ECOG < 
$3(216,58.69 \%)$ and ECOG $\geq 3(152,41.31 \%)$. Patients with poor nutritional status accounted for the vast majority of the study patients $(289,78.53 \%)$.

In cohort 2, there was a similar number of males $(55,52.38 \%)$ and females $(50,47.62 \%)$. Patients with stage IV disease $(89,84.76 \%)$ and gastrointestinal tumors $(58,55.24 \%)$ accounted for the vast majority of cases. Unlike cohort 1 , cohort 2 had more patients who received palliative chemoradiotherapy (PCR) than best supportive care (BSC) (54 [51.43\%] vs. 51 [48.57\%]). There were far more patients with ECOG < 3 (79, $75.24 \%)$ than ECOG $\geq 3(26,24.76 \%)$. The number of patients with good nutritional status $(41,39.05 \%)$ was less than the number with poor nutritional status $(64,60.95 \%)$.

Table 1

Comparisons of baseline clinicopathological features based on $\mathrm{Hb}$ in cohort $1(\mathrm{~N}=368)$ 


\begin{tabular}{|c|c|c|c|c|}
\hline \multirow{2}{*}{$\begin{array}{l}\text { Clinicopathological } \\
\text { features }\end{array}$} & \multirow[t]{2}{*}{$\mathrm{N}(\%)$} & \multicolumn{2}{|l|}{$\mathrm{Hb}$} & \multirow[t]{2}{*}{$P$ value } \\
\hline & & $\mathrm{HHb}(\mathrm{n}=236)$ & LHb $(n=132)$ & \\
\hline Age (Mean \pm SD) & $\begin{array}{l}368 \\
(63.56 \pm 12.881)\end{array}$ & $64.42 \pm 12.423$ & $62.04 \pm 13.576$ & 0.09 \\
\hline Gender & & & & 0.008 \\
\hline Male & $201(54.62 \%)$ & $141(59.74 \%)$ & $60(45.45 \%)$ & \\
\hline Female & $167(45.38 \%)$ & $95(40.26 \%)$ & $72(54.55 \%)$ & \\
\hline \multicolumn{5}{|l|}{ Tumor stage } \\
\hline III & $29(7.88 \%)$ & $20(8.47 \%)$ & $9(6.82 \%)$ & 0.572 \\
\hline IV & $339(92.12 \%)$ & $216(91.53 \%)$ & 123(93.18\%) & \\
\hline Primary tumor site & & & & 0.709 \\
\hline Gastrointestinal tumors & $189(51.36 \%)$ & $118(50 \%)$ & $71(53.79 \%)$ & \\
\hline Thoracic cancers & $83(22.55 \%)$ & $56(23.73 \%)$ & $27(20.45 \%)$ & \\
\hline Urogenital neoplasms & $60(16.31 \%)$ & $36(15.25 \%)$ & $24(18.18 \%)$ & \\
\hline Head and neck cancers & $17(4.62 \%)$ & $12(5.08 \%)$ & $5(3.79 \%)$ & \\
\hline Other tumors & $19(5.16 \%)$ & $14(5.94 \%)$ & $5(3.79 \%)$ & \\
\hline Palliative care & & & & 0.004 \\
\hline PCR & $93(25.27 \%)$ & $71(30.08 \%)$ & $22(16.67 \%)$ & \\
\hline BSC & $275(74.73 \%)$ & $165(69.92 \%)$ & 110(83.33\%) & \\
\hline Family history & & & & 0.372 \\
\hline No & $260(70.65 \%)$ & 163(69.07\%) & $97(73.48 \%)$ & \\
\hline Yes & 108(29.35\%) & $73(30.93 \%)$ & $35(26.52 \%)$ & \\
\hline ECOG & & & & 0.584 \\
\hline$<3$ & $216(58.69 \%)$ & $141(59.75 \%)$ & $75(56.82 \%)$ & \\
\hline 33 & $152(41.31 \%)$ & $95(40.25 \%)$ & $57(43.18 \%)$ & \\
\hline Comorbidity & & & & 0.998 \\
\hline No & $223(60.59 \%)$ & $143(60.59 \%)$ & $80(60.61 \%)$ & \\
\hline Yes & 145(39.41\%) & $93(39.41 \%)$ & $52(39.39 \%)$ & \\
\hline Nutritional status & & & & 0.481 \\
\hline Normal & $79(21.47 \%)$ & $48(20.34 \%)$ & $31(23.49 \%)$ & \\
\hline
\end{tabular}


Abbreviations: $\mathrm{Hb}$, hemoglobin; SD, standard deviation; LHb, low Hb (pretreatment $\mathrm{NC} £ 100$ ); $\mathrm{HHb}$, high $\mathrm{Hb}$ (pretreatment NC > 100); BSC, best supportive care; PCR, palliative chemoradiotherapy; ECOG, Eastern Cooperative Oncology Group

Table 2

Comparisons of baseline clinicopathological features based on $\mathrm{Hb}$ in cohort $2(\mathrm{~N}=105)$ 


\begin{tabular}{|c|c|c|c|c|}
\hline \multirow{2}{*}{$\begin{array}{l}\text { Clinicopathological } \\
\text { features }\end{array}$} & \multirow[t]{2}{*}{$\mathrm{N}(\%)$} & \multicolumn{2}{|l|}{$\mathrm{Hb}$} & \multirow{2}{*}{$\begin{array}{l}P \\
\text { value }\end{array}$} \\
\hline & & Descending & Ascending & \\
\hline Age (Mean \pm SD) & $105(62.92 \pm 12.012)$ & $63(62.11 \pm 11.278)$ & $42(64.14 \pm 13.081)$ & 0.398 \\
\hline Gender & & & & 0.69 \\
\hline Male & $55(52.38 \%)$ & $34 \llbracket 53.97 \% \rrbracket$ & $21 \otimes 50 \% \bigotimes$ & \\
\hline Female & $50(47.62 \%)$ & $29 \llbracket 46.03 \% \rrbracket$ & $21 \otimes 50 \% \bigotimes$ & \\
\hline Tumor stage & & & & 0.438 \\
\hline III & 16ه15.24\%】 & $11 \otimes 17.46 \% \bigotimes$ & $5 \rrbracket 11.91 \% \rrbracket$ & \\
\hline IV & $89 \rrbracket 84.76 \% \rrbracket$ & $52 \varangle 82.54 \% \rrbracket$ & $37 \rrbracket 88.09 \% \rrbracket$ & \\
\hline Primary tumor site & & & & 0.653 \\
\hline Gastrointestinal tumors & $58 \rrbracket 55.24 \% \bigotimes$ & $33 \llbracket 52.38 \% \rrbracket$ & $25 \rrbracket 59.53 \% \bigotimes$ & \\
\hline Thoracic cancers & $14 \rrbracket 13.33 \% \rrbracket$ & $8 \rrbracket 12.69 \% \rrbracket$ & $6 \rrbracket 14.28 \% \rrbracket$ & \\
\hline Urogenital neoplasms & $24 \rrbracket 22.86 \% \rrbracket$ & $17 \rrbracket 26.99 \% \rrbracket$ & $7 \rrbracket 16.67 \% \rrbracket$ & \\
\hline Head and neck neoplasm & $6 \rrbracket 5.71 \% \rrbracket$ & $4 \llbracket 6.34 \% \rrbracket$ & $2 \rrbracket 4.76 \% \rrbracket$ & \\
\hline Other tumors & $3 \rrbracket 2.86 \% \rrbracket$ & $1 \otimes 1.59 \% \rrbracket$ & $2 \rrbracket 4.76 \% \rrbracket$ & \\
\hline Palliative care & & & & 0.3 \\
\hline PCR & $54 \rrbracket 51.43 \% \rrbracket$ & $35 \llbracket 55.56 \% \rrbracket$ & $19 ه 45.24 \% \bigotimes$ & \\
\hline BSC & $51 \rrbracket 48.57 \% \rrbracket$ & $28 \llbracket 44.44 \% \rrbracket$ & $23 \llbracket 54.76 \% \rrbracket$ & \\
\hline Family history & & & & 0.226 \\
\hline No & $73 \rrbracket 69.52 \% \rrbracket$ & $41 \llbracket 65.08 \% \rrbracket$ & $32 \otimes 76.19 \% \rrbracket$ & \\
\hline Yes & $32 \bowtie 30.43 \% \rrbracket$ & $22 \llbracket 34.92 \% \rrbracket$ & $10 \rrbracket 23.81 \% \rrbracket$ & \\
\hline ECOG & & & & 0.854 \\
\hline$<3$ & $79 \rrbracket 75.24 \% \rrbracket$ & $47 \rrbracket 74.60 \% \rrbracket$ & $32 \bigotimes 76.19 \% \rrbracket$ & \\
\hline${ }^{3} 3$ & $26 \rrbracket 24.76 \% \rrbracket$ & $16 \rrbracket 25.40 \% \rrbracket$ & $10 \rrbracket 23.81 \% \rrbracket$ & \\
\hline Comorbidity & & & & 0.218 \\
\hline No & $65 \rrbracket 61.90 \% \rrbracket$ & $42 \llbracket 66.67 \% \rrbracket$ & $23 \otimes 54.76 \% \bigotimes$ & \\
\hline Yes & $40 \rrbracket 38.10 \% \rrbracket$ & $21 \varangle 33.33 \% \rrbracket$ & $19 \varangle 45.24 \% \rrbracket$ & \\
\hline Nutritional status & & & & 0.288 \\
\hline Normal & $41 \rrbracket 39.05 \% \rrbracket$ & $22 \rrbracket 34.92 \% \rrbracket$ & $19 \rrbracket 45.24 \% \rrbracket$ & \\
\hline
\end{tabular}


Abbreviations: $\mathrm{Hb}$, hemoglobin; SD, standard Deviation; BSC, best supportive care; PCR, palliative chemoradiotherapy; ECOG, Eastern Cooperative Oncology Group

\section{Determination of the $\mathrm{Hb}$ cut-off value}

In the clinical environment, it is common to convert continuous variables to binary variables. In this study, the patients were divided into "high" and "low" groups, which was convenient for diagnostic or prognostic predictions. However, for patients with advanced cancer, the accepted clinical threshold was not available. Using $\mathrm{X}$-tile based on a minimum $\mathrm{p}$-value algorithm ${ }^{[10]}$, the optimal cut-off value for $\mathrm{Hb}$ was determined to be $100 \mathrm{~g} / \mathrm{L}$. Therefore, the patients with HB levels greater than $100 \mathrm{~g} / \mathrm{L}$ were assigned to the high $\mathrm{HB}(\mathrm{HHB})$ group and the patients with $\mathrm{HB}$ levels less than or equal to $100 \mathrm{~g} / \mathrm{L}$ were part of the low HB (LHB) group.

\section{Association of $\mathrm{Hb}$ with clinicopathological features}

The relationships between the clinicopathological features and $\mathrm{Hb}$ (low vs. high) for cohort 1 are shown in Table 1. There were no differences in age, tumor site, family history, ECOG, nutritional status, or comorbidity between the HHB and LHB groups. In contrast, palliative treatment and gender were significantly associated with $\mathrm{Hb}$. The characteristics of cohort 2 are shown in Table 2, where patients were divided into descending (changes $<0$ ) and ascending (changes $>0$ ) groups. There was no significant difference in the proportion of patients with specific clinicopathological features between the two groups.

\section{Association of $\mathrm{Hb}$ with $\mathrm{OS}$}

Patients in cohort 1 were divided into $\mathrm{HHb}$ and LHb groups. The survival time for the HHB group was significantly longer than that of the LHB group (median survival time: 84 days [95\% Cl 61-107] vs. 41 days [95\% $\mathrm{Cl} 31-51), P<0.001$ ) (Figure 2). Multivariate Cox proportional hazard model showed that tumor stage, ECOG score, palliative treatment, and $\mathrm{Hb}$ were independent prognostic factors for OS. After controlling for important confounding variables, HB was still significantly correlated with OS (HHB vs. LHB, HR 0.733, 95\% Cl 0.558-0.909) (Table 3).

We evaluated cohort 2 to verify the prognostic significance of dynamic changes in $\mathrm{Hb}$ by dividing into ascending and descending groups. We found that patients who changed from the $\mathrm{HHb}$ group pretreatment to $\mathrm{LHb}$ after the second admission had the worst $\mathrm{OS}(\mathrm{LHb} \rightarrow \mathrm{LHb}$ median survival time [109, $95 \% \mathrm{Cl} 49-169]$ vs. LHb $\rightarrow \mathrm{HHb}$ median survival time [104, 95\% $\mathrm{Cl} 0-276]$ vs. $\mathrm{HHb} \rightarrow \mathrm{LHb}$ median survival time [68, 95\% C: $45-90]$ vs. $\mathrm{HHb} \rightarrow \mathrm{HHb}$ median survival time [234, 95\% $\mathrm{Cl} 168-300], P \otimes 0.013)$ 
(Figure 3). The related subgroups were further analyzed and significant results were obtained after adjusting for demographic and disease-specific factors (Table 4).

Table 3

Multivariate Cox regression analysis for $\mathrm{Hb}(\mathrm{N}=368)$

\begin{tabular}{|c|c|c|}
\hline \multirow[t]{2}{*}{ Prognostic factors } & \multicolumn{2}{|l|}{ Hb Model ${ }^{\#}$} \\
\hline & Adjusted HR (95\% Cl) & $P$ value \\
\hline Gender (male vs. female) & $0.834 \llbracket 0.634-1.034 \rrbracket$ & 0.09 \\
\hline Age & $0.998 \rrbracket 0.988-1.008 \rrbracket$ & 0.635 \\
\hline Primary tumor site & $1.008 \bowtie 0.376-1.640 \rrbracket$ & 0.156 \\
\hline Tumor stage (IV vs. III) & 7.089(2.710-11.469) & $\varangle 0.001$ \\
\hline ECOG (<3 vs. $\geq 3)$ & $0.606(0.461-0.751)$ & 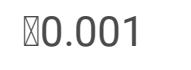 \\
\hline Nutritional status (normal vs. abnormal) & 1.068(0.763-1.373) & 0.879 \\
\hline Palliative care (PCR vs. BSC) & $0.573(0.401-0.745)$ & 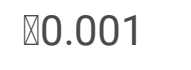 \\
\hline Comorbidity (yes vs. no) & $0.855(0.640-1.071)$ & 0.151 \\
\hline Family history (yes vs. no) & $1.217(0.915-1.520)$ & 0.203 \\
\hline HHb vs. LHb & $0.733(0.558-0.909)$ & 0.006 \\
\hline \multicolumn{3}{|c|}{$\begin{array}{l}\text { Abbreviations: Hb, hemoglobin; HR, hazard ratio; } \mathrm{Cl} \text {, confidence interval; } \mathrm{LHb} \text {, low } \mathrm{Hb} \text { (pretreatment } \leq \\
\text { 100); HHb, high Hb (pretreatment > 100); BSC, best supportive care; PCR, palliative } \\
\text { chemoradiotherapy; ECOG, Eastern Cooperative }\end{array}$} \\
\hline
\end{tabular}

\section{Oncology Group.}

\#The potential prognostic factors used for adjustment in the Cox regression model controlling for gender; age; primary tumor site; tumor stage; ECOG; nutrient status; palliative care; comorbidity; family history.

Table 4

Adjusted HRs for overall survival stratified by changes in $\mathrm{Hb}$ in cohort $2(\mathrm{~N}=105)$ 


\begin{tabular}{|c|c|c|}
\hline \multirow[t]{2}{*}{$\mathrm{Hb}$} & \multicolumn{2}{|l|}{ Hb Model ${ }^{\#}$} \\
\hline & Adjusted HR (95\% Cl) & $P$ value \\
\hline Ascending & \multicolumn{2}{|l|}{ Reference } \\
\hline Descending & $0.684(0.366-1.002)$ & 0.051 \\
\hline $\mathrm{LHb} \rightarrow \mathrm{LHb}$ & \multicolumn{2}{|l|}{ Reference } \\
\hline $\mathrm{LHb} \rightarrow \mathrm{HHb}$ & $1.841(0.211-3.472)$ & 0.828 \\
\hline $\mathrm{HHb} \rightarrow \mathrm{LHb}$ & $4.003(1.421-6.585)$ & 0.004 \\
\hline $\mathrm{HHb} \rightarrow \mathrm{HHb}$ & $0.701(0.293-1.109)$ & 0.098 \\
\hline \multicolumn{3}{|c|}{$\begin{array}{l}\text { Abbreviations: Hb, hemoglobin; HR, hazard ratio; } \mathrm{Cl} \text {, confidence interval; LHb, low } \mathrm{Hb} \text { (pretreatment } £ \\
\text { 100); HHb, high } \mathrm{Hb} \text { (pretreatment }>100 \text { ). } \\
\text { \#The potential prognostic factors used for adjustment in the Cox regression model controlling for } \\
\text { gender, age, primary tumor site, tumor stage, ECOG, nutrient status, palliative care, comorbidity, and } \\
\text { family history. }\end{array}$} \\
\hline
\end{tabular}

\section{Discussion}

Although tremendous progress has been made in the treatment of cancer, the likelihood of treatment failure for patients with advanced disease remains high. Survival assessment is necessary for risk stratification and further clinical decision-making. However, no significant biomarkers have been widely used in survival assessment. Hemoglobin is an indicator measured in almost all hospitals in China. In our study, we evaluated the prognostic impact of $\mathrm{Hb}$ on patients with advanced cancer.

This study is the first to investigate the dynamic changes of hemoglobin in patients with advanced cancer in the palliative treatment environment. We established a Hb cut-off value of $100 \mathrm{~g} / \mathrm{L}$ and found that patients with a low $\mathrm{Hb}$ had a poorer survival time than patients with high $\mathrm{Hb}$ with advanced cancer. Furthermore, patients that transitioned from high $\mathrm{Hb}$ levels to low $\mathrm{Hb}$ levels between the first and second hospital admission were at a greater risk of a poor prognosis. In conclusion, pretreatment and dynamic peripheral hemoglobin levels represent convenient and intuitive markers to predict aggressiveness in patients with advanced cancer.

Ludwig et al. ${ }^{[11]}$ found that the incidence of anemia in 11,453 patients with various cancers was $41 \%$. In patients with advanced tumors and those receiving radiotherapy or chemotherapy, the incidence of anemia is as high as $90 \%{ }^{[12]}$, especially in patients with more aggressive tumors. This finding may be related to the complex interactions between the immune system, tumor microenvironment, and cancer cells ${ }^{[13,14]}$. In general, cancer-related anemia has various causes, including blood loss, functional iron deficiency, erythropoietin deficiency due to kidney disease, and the relationship between cancer and bone marrow $^{[15]}$. Tumor cells can activate macrophages, promote the secretion of inflammatory factors (e.g., gamma-interferon, IL-1, and tumor necrosis factor), which can reduce $\mathrm{Hb}$ concentrations by altering the 
hematopoietic environment ${ }^{[16,17]}$, inhibiting erythropoiesis and erythropoietin, and impairing the erythropoietin response of erythroid progenitor cells ${ }^{[18,19]}$. In addition, bone marrow failure is common in patients with bone metastases and leads to low $\mathrm{Hb}$ levels ${ }^{[20]}$. Patients at later tumor stages and greater tumor burden have more inflammatory factors secreted by tumor cells, a higher incidence of anemia, and a worse prognosis ${ }^{[21]}$. Hypoxia is an important link in the occurrence and development of malignant tumors ${ }^{[22]}$, while anemia will aggravate the degree of hypoxia in tumor tissues and affect the stability of chemotherapeutic drugs in the process of diffusion. Moreover, the production of oxygen-free radicals reduces the damaging effect of chemotherapeutic drugs on the DNA of tumor cells, thus reducing drug efficacy ${ }^{[23]}$. Tissue oxygenation disorders stimulate the high expression of angiogenic factors, which promotes the occurrence and development of tumors, reduces drug sensitivity, increases the probability of blood transfusion after chemotherapy, damages organ function, reduces the quality of life, and increases postoperative mortality.

A meta-analysis involving 60 studies showed that anemia increased the overall risk of death in tumor patients by $65 \%[24]$. Previous studies showed that anemia might impact the biological characteristics and prognosis of tumor patients, reduced the response to chemotherapy, and promoted tumor recurrence and metastasis, which is an independent risk factor of poor prognosis ${ }^{[25]}$. Studies have reported that anemia may or may not be a bad prognostic factor in patients with advanced gastric cancer ${ }^{[26,27]}$. These results may be related to the inconsistent reference criteria for anemia used in the different studies. The U.S. National Cancer Institute $(\mathrm{NCl})$ and the World Health Organization (WHO) define their own anemia diagnosis and classification standard. The Chinese Society of Clinical Oncology (notes) is based on clinical practice and the treatment of cancer-related anemia classification ${ }^{[28]}$. The different standards in defining the normal $\mathrm{Hb}$ range and anemia are different for these entities based on slightly different severity classifications. Therefore, to unify the criteria, we first established a threshold value for $\mathrm{Hb}$ as a tool to predict the prognosis of patients with advanced cancer.

Although this study provides important insights into the relationship between $\mathrm{Hb}$ levels and the prognosis of patients with advanced cancer, it has some limitations. This study was limited to a retrospective single-center design with inherent limitations, such as selection bias, possible confounders, and relatively low sample size. Therefore, larger prospective multi-institutional studies with more clinical markers are needed to validate the relationship between $\mathrm{Hb}$ and poor prognosis. Overall, we demonstrated for the first time that $\mathrm{Hb}$ is a strong independent predictor of shortened survival after palliative treatment in patients with advanced cancer. As an easily accessible and inexpensive biomarker, $\mathrm{Hb}$ deserves further research into future clinical practice for risk stratification and may enable more accurate clinical decision-making for patients with advanced cancer.

\section{Conclusions}

In conclusion, in palliative treatment, hemoglobin can be used as a stratification factor to determine the prognosis of advanced cancer patients. Patient survival time can be estimated from the changes after 


\section{Declarations}

\section{Author contributions}

All authors made substantial contributions to one or more of the following: study conception and design (XHL, WWC); acquisition of data or analysis (XHL, WWZ, MLC, XLG, MHL, ZZ, CYZ); interpretation of data (XHL, WWZ, CHB, ZZ). XHL and CHB drafted the article, and all other authors contributed to critically revising the article for important intellectual content. All authors provided their final approval of the version to be published.

\section{ACKNOWLEDGMENTS AND FUNDING}

We are grateful to the participating patients, their families, and all our co-investigators who contributed to this study. This work was supported by the National Natural Science Foundation of China (81400810, 81101762, and 11371100).

\section{CONFLICTS OF INTEREST}

The authors declare no conflicts of interest.

\section{References}

1. Dong ST, Butow PN, Costa DS, Lovell MR, Agar M. Symptom clusters in patients with advanced cancer: a systematic review of observational studies. J Pain Symptom Manag. 2014;48(3):411-50.

2. Smith TJ, Temin S, Alesi ER, et al. American Society of Clinical Oncology provisional clinical opinion: the integration of palliative care into standard oncology care. J Clin Oncol. 2012;30(8):880-7.

3. Finlay E, Casarett D. Making difficult discussions easier: using prognosis to facilitate transitions to hospice. CA Cancer J Clin. 2009;59(4):250-63

4. Lambert SD, Harrison JD, Smith E, et al. The unmet needs of partners and caregivers of adults diagnosed with cancer: a systematic review. BMJ Support Palliat Care. 2012;2(3):224-30

5. Amano K, Maeda I, Shimoyama S, et al. The accuracy of Physicians' clinical predictions of survival in patients with advanced cancer. J Pain Symptom Manag. 2015;50(2):139-46.

6. Zhang YH, Lu Y, Lu H, et al. Pre-treatment hemoglobin levels are an independent prognostic factor in patients with non-small cell lung cancer. Mol Clin Oncol. 2018; 9: 44-49

7. Weiss G, Goodnough LT. Anemia of chronic disease. The New England journal of medicine. 2005; 352: $1011-23$ 
8. Kondrup J, Allison SP, Elia M, Vellas B, Plauth M. ESPEN guidelines for nutrition screening 2002. Clin Nutr. 2003;22(4):415-21

9. Zhao W, Wu Z, Li Y,et al. Pretreatment neutrophil-tolymphocyte ratio and its dynamic changes are associated with the overall survival in advanced cancer patients undergoing palliative care. Sci Rep. 2016; 6:31394

10. Camp RL, Dolled-Filhart M, Rimm DL. X-tile: a new bio-informatics tool for biomarker assessment and outcome-based cut-point optimization. Clin Cancer Res. 2004; 10:7252-7259.

11. Ludwig H,Van Belle S,Barrett-LeeP,et al.The European Cancer Anemia Survey(ECAS):a large,multinational,prospective survey defining the prevalence,incidence,and treatment of anemia in cancer patients[J].Eur J Cancer,2004,40(15):2293-2306.

12. Glaspy J,Cavill I.Role of iron in optimizing responses of ane-mic cancer patients to erythropoietin [J].Oncology,1999,13(4): 461-473.

13. Mercadante S, Gebbia V, Marrazzo A, et al. Anaemia in cancer: pathophysiology and treatment. Cancer Treat Rev. 2000; 26: 303-311

14. Birgegard G, Aapro MS, Bokemeyer C, et al. Cancer-related anemia: pathogenesis, prevalence and treatment. Oncology. 2005; 68 Suppl 1: 3-11.

15. Xia L, Guzzo TJ. Preoperative Anemia and Low Hemoglobin Level Are Associated With Worse Clinical Outcomes in Patients With Bladder Cancer Undergoing Radical Cystectomy: A Meta-Analysis. Clin Genitourin Cancer.2017; 15: 263-272.e4.

16. Banzet $S$, Sanchez $H$, Chapot $R$, et al. Interleukin- 6 contributes to hepcidin mRNA increase in response to exercise. Cytokine. 2012; 58: 158-161.

17. Sun CC, Vaja V, Babitt JL, et al. Targeting the hepcidin-ferroportin axis to develop new treatment strategies for anemia of chronic disease and anemia of inflammation. Am J Hematol. 2012; 87: 392-400.

18. Wu Y, Antony S, Meitzler JL, et al. Molecular mechanisms underlying chronic inflammationassociated cancers. Cancer Lett. 2014; 345: 164-173.

19. Aapro M, Jelkmann W, Constantinescu SN, et al. Effects of erythropoietin receptors and erythropoiesis-stimulating agents on disease progression in cancer. $\mathrm{Br} \mathrm{J}$ Cancer. 2012; 106: 1249 1258.

20. Tefferi A, Hudgens S, Mesa R, et al. Use of the Functional Assessment of Cancer Therapy-anemia in persons with myeloproliferative neoplasm-associated myelofibrosis and anemia. Clin Ther. 2014; 36: 560-566.

21. Pan J, Qin S, Chen Y, et al.Analysis of clinical factors in the conversion of colorectal cancer with simultaneous liver metastasis to resectable[J].Chin Clin Oncol,2019,24(1):44-48.

22. Li S, Meng W, Guan Z, et al. The hypoxia-related signaling pathways of vasculogenic mimicry in tumor treatment. Biomed Pharmacother. 2016; 80:127-135. 
23. Chen Y,Wang YR,Zhang Q, et al. Relationship between tumor-associated anemia and prognosis in patients with advanced gastric cancer [J]. Academic Journal of Chinese PLA Medical School, 2015, 36(4): 351-354.

24. Caro JJ, Salas $M$, Ward A, et al. Anemia as an independent prognostic factor for survival in patients with cancer: a systemic, quantitative review. Cancer. 2001;91(12):2214-2221.

25. Qiu MZ, Xu RH, Ruan DY, et al. $11 \% 4 @$ ncidence of anemia, leukocytosis, and thrombocytosis in patients with solid tumors in China[J]. Tumour Biol, 2010, 31(6): 633-641

26. Mohri Y,Tanaka K,Ohi M,et al.Prognostic significance of host and tumor related factors in patients with gastric cancer[J]. World J Surg,2010,34(2):285-290.

27. Kanagavel D,Pokataevl A,Fedyanin MY,et al. A prognostic model in patients treatsd for metastatic gastric cancer with second-line chemotherapy[J].Ann Oncol,2010,21(9):1779-1785.

28. Ma J,Wang JJ,Zhang L,et al. Clinical Practice Guidelines for Tumor-associated Anemia (20152016). Chinese Journals of Practical Medicine. 2015;(11):921-930

\section{Figures}




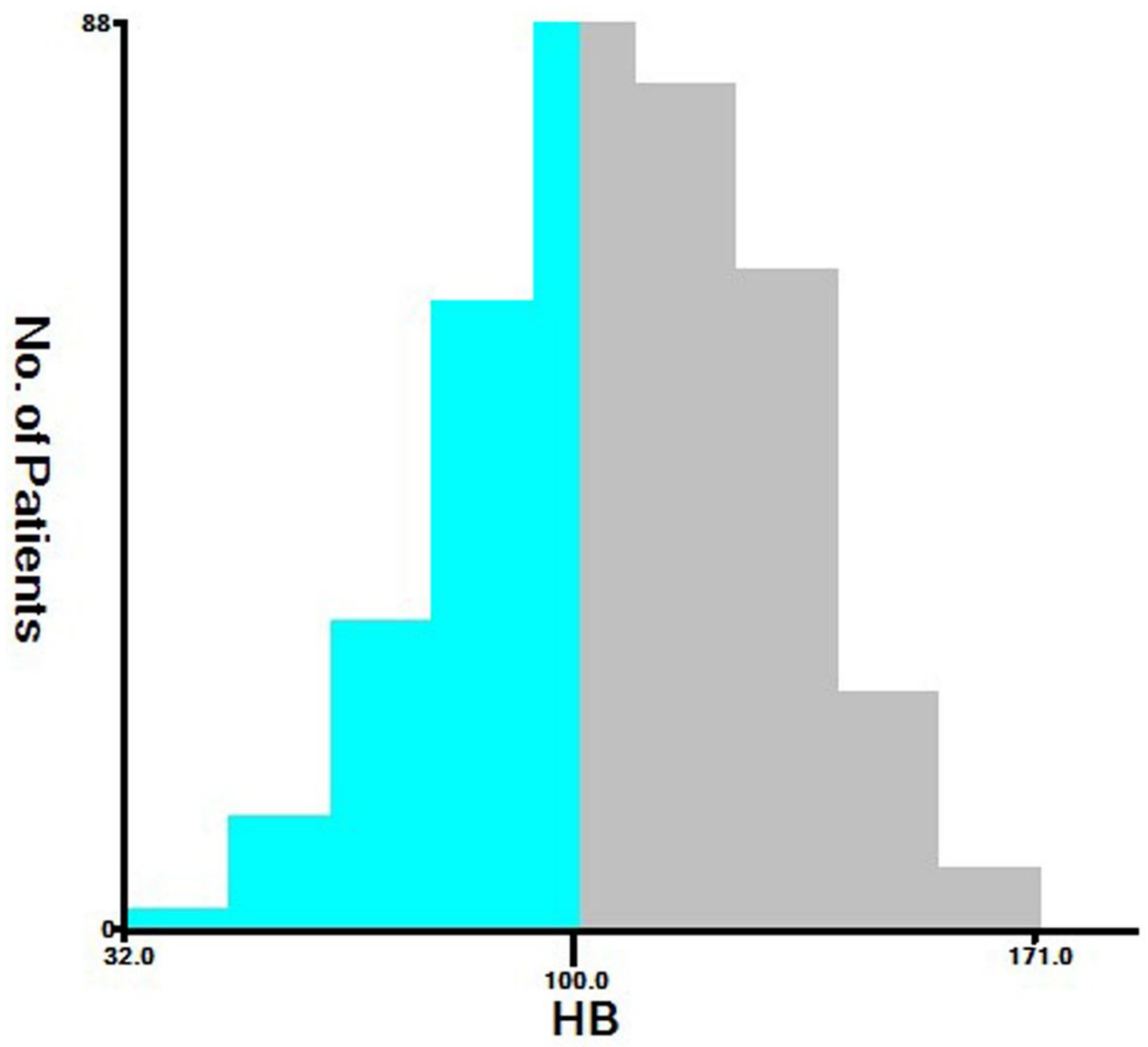

Figure 1

X-tile analysis was performed to determine the optimal cut-off values using the data of cohort 1 . The optimal cut-off value for HB was $100(x 2=15.85, P=0.0026)$. 


\section{Strata $+\mathrm{LHb}+\mathrm{HHb}$}

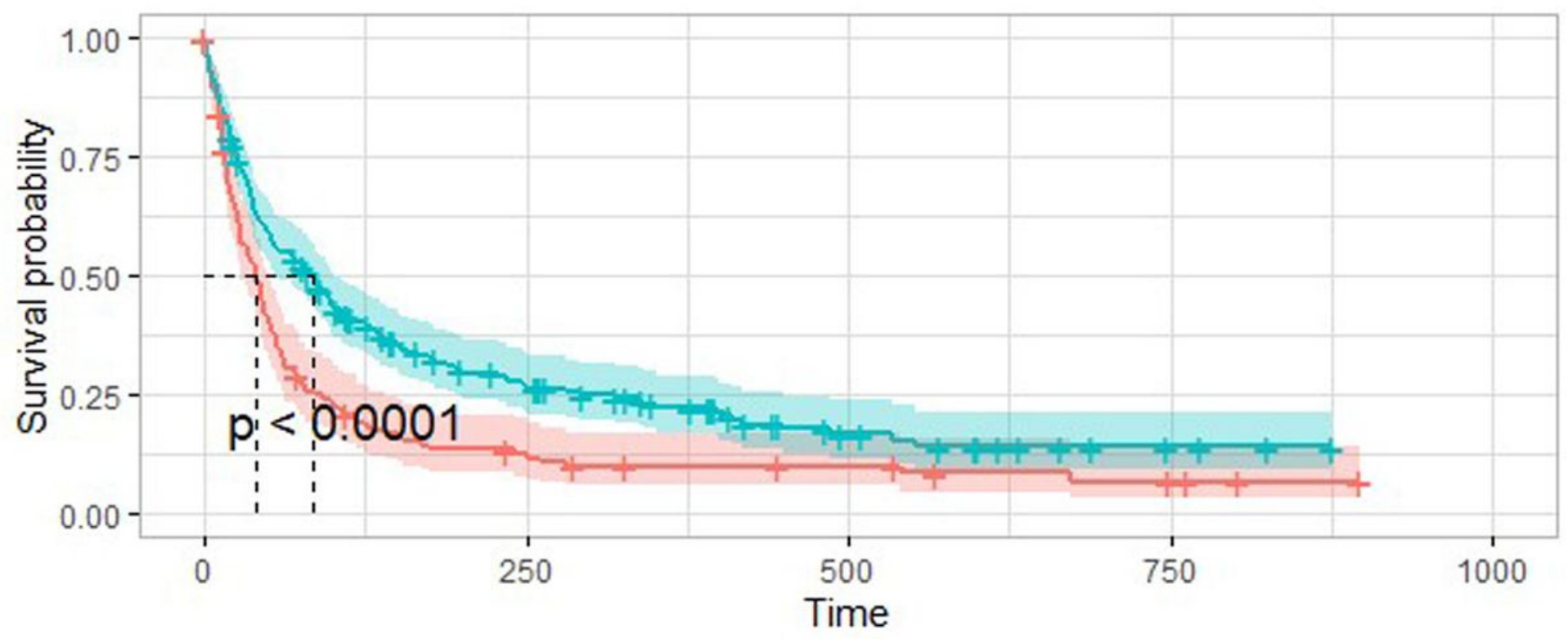

Number at risk
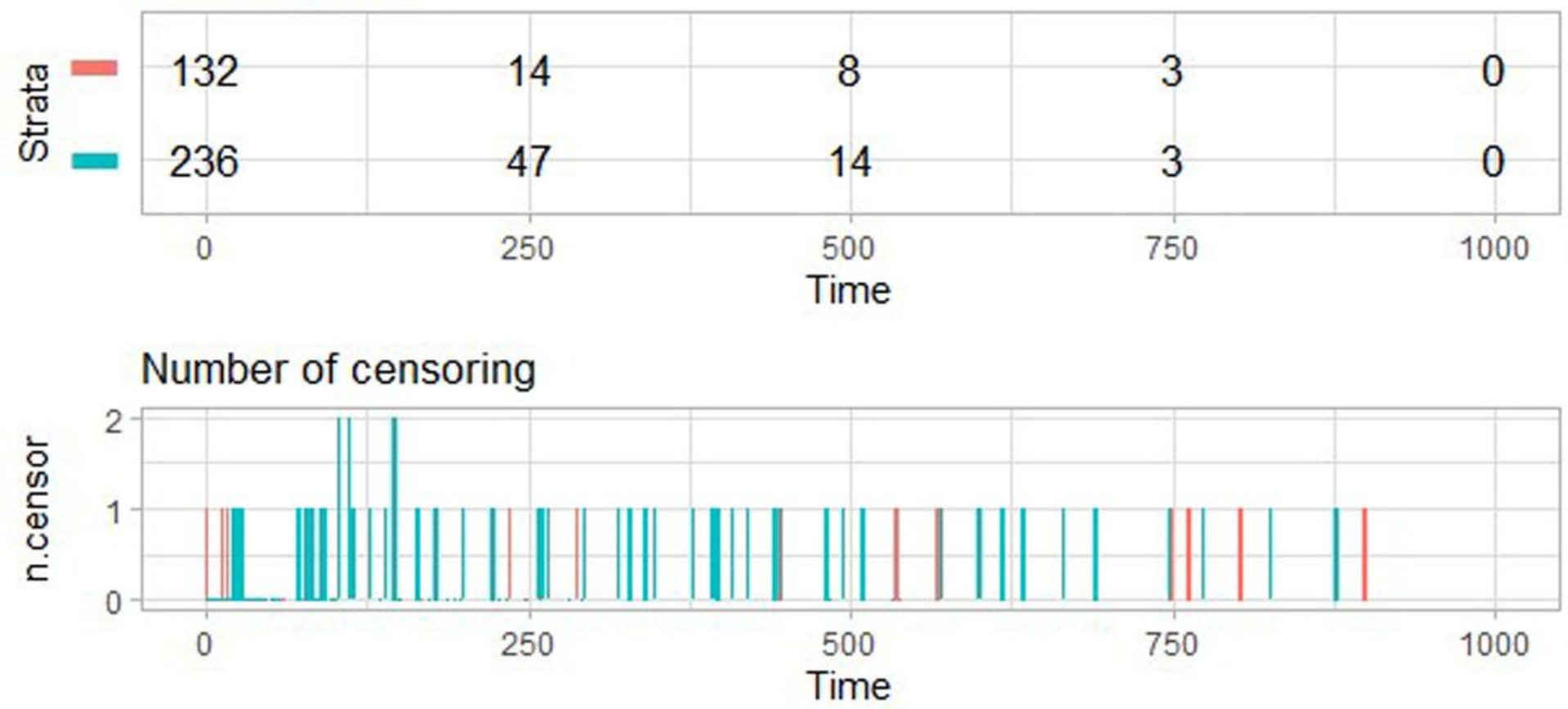

Figure 2

Overall survival of patients under palliative care stratified by pretreatment $\mathrm{Hb}$ (cohort 1 ). Abbreviations: $\mathrm{LHb}$, low $\mathrm{Hb}$ (pretreatment $\leq 100$ ); HHb, high $\mathrm{Hb}$ (pretreatment > 100) 


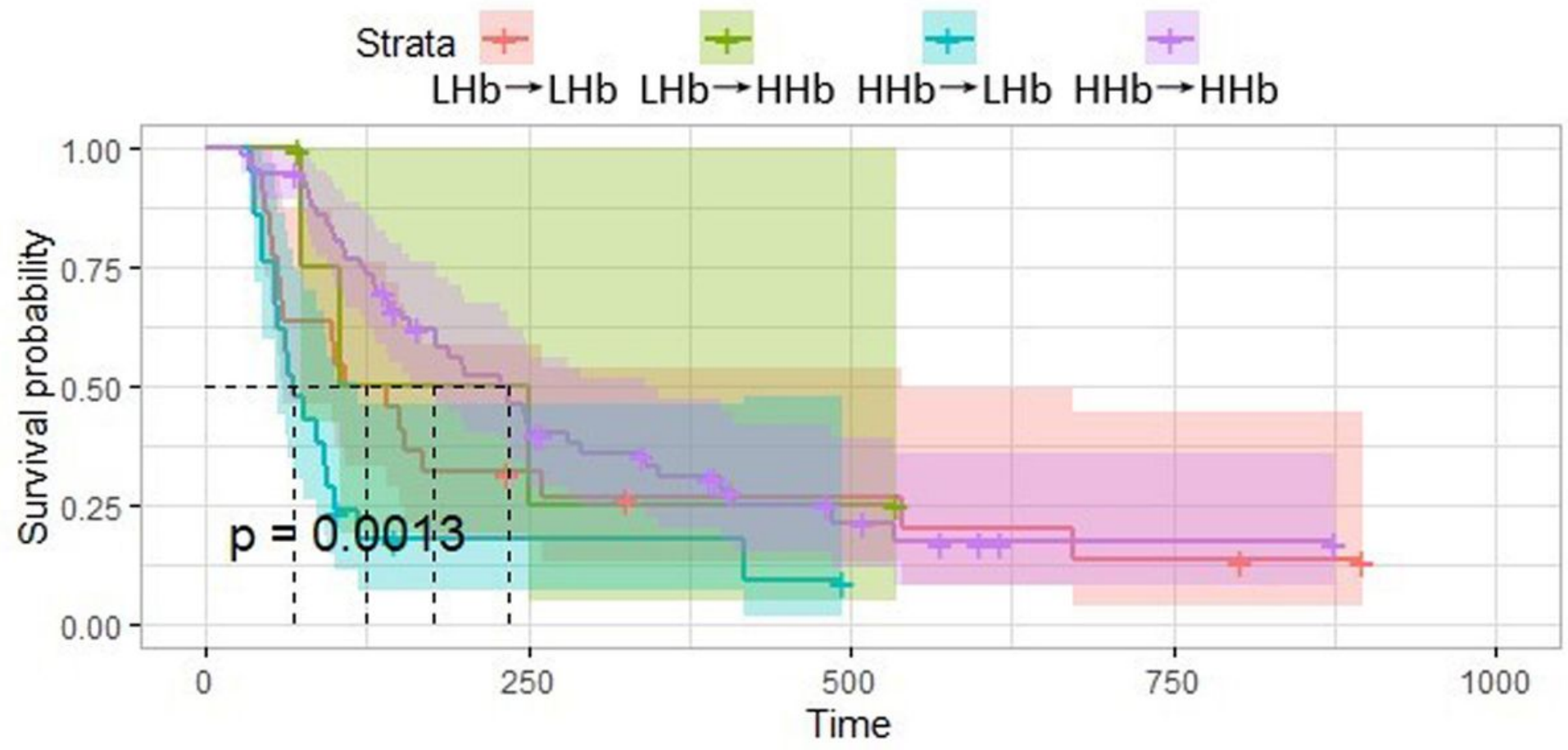

Number at risk

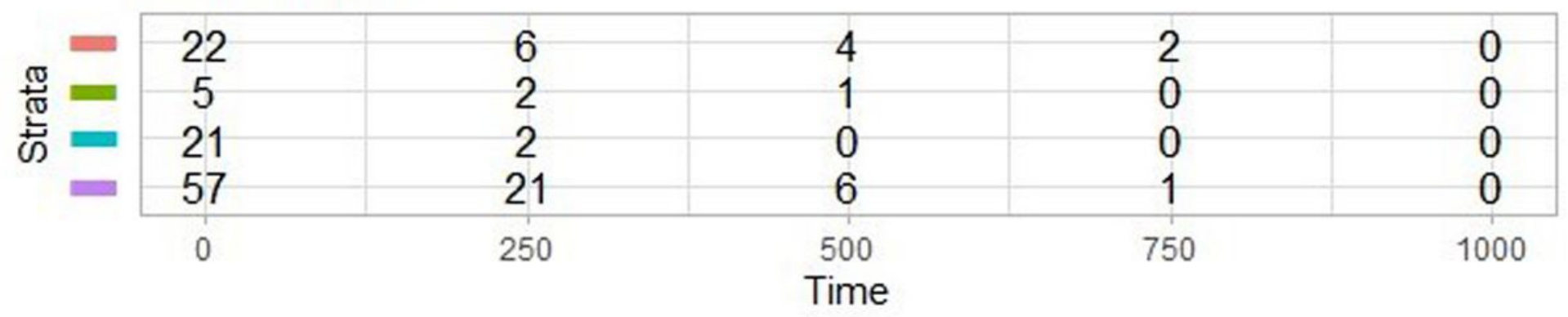

Number of censoring

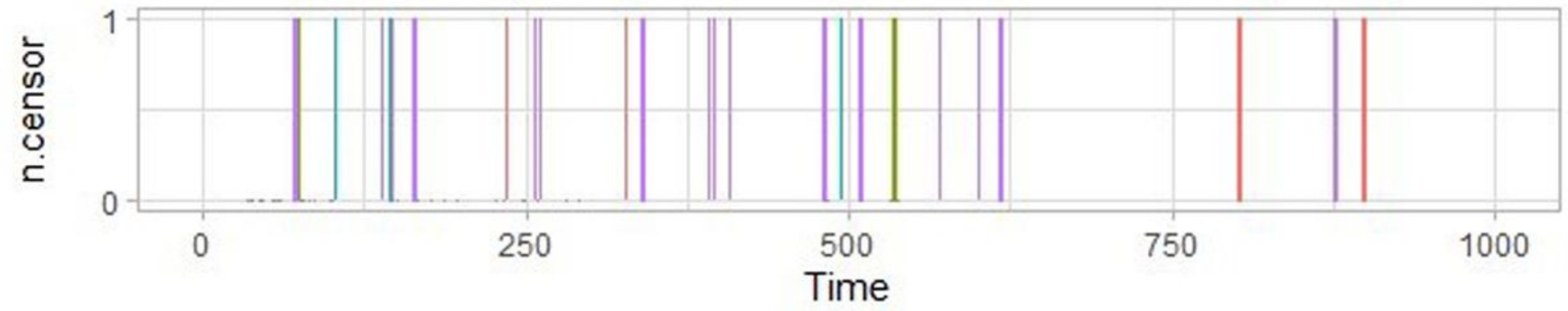

Figure 3

Overall survival of patients under palliative care stratified by changes in $\mathrm{Hb}$ (cohort 2). Abbreviations: $\mathrm{LHb}$, low $\mathrm{Hb}$ (pretreatment $\leq 100$ ); $\mathrm{HHb}$, high $\mathrm{Hb}$ (pretreatment > 100) 